\title{
Factibilidad instrumental de la conformación de redes productivas en las pyme: el caso del sector del calzado en la provincia de Tungurahua
}

\author{
María del Carmen Gómez Romo \\ Pontificia Universidad Católica del Ecuador, Sede Ambato (Ecuador) \\ mariadgomez@uta.edu.ec \\ Andrés Francisco López Gómez \\ Pontificia Universidad Católica del Ecuador, Sede Ambato (Ecuador) \\ andresflopez@uta.edu.ec
}

Fecha de presentación: 13 diciembre de 2016 • Fecha de aceptación: 25 de febrero de 2018 Artículo de investigación 


\section{Resumen}

Esta investigación presenta un caso aplicado a pequeñas y medianas empresas del sector del calzado en la provincia de Tungurahua. El objetivo es determinar la factibilidad instrumental o cooperación más provechosa para la conformación de una red productiva. Se indaga entre los empresarios los factores críticos, su importancia y grados de posibles complementaciones para identificar los aspectos que motivan la conformación de la red que se ven reflejados en los índices de cooperación provechosa (ICP). Finalmente, se establece que el ICP más alto corresponde al factor producción que es por donde se recomienda iniciar acciones para la conformación de la red.

Palabras clave: asociatividad, redes productivas, pyme, sector calzado, Ecuador.

JEL: M1 Administración de Negocios; D7 Análisis de la toma de decisiones colectiva.

\section{Abstract}

This research presents applied case to small and medium companies in the footwear sector in the province of Tungurahua. The objective is to determine the most profitable instrumental feasibility or cooperation for the formation of a productive network. Entrepreneurs are asked about the critical factors, their importance and degrees of possible complements to identify the aspects that motivate the conformation of the network that are reflected in the indexes of beneficial cooperation (IBC). Finally, it is established that the highest IBC corresponds to the production factor, where it is recommended to initiate actions for the conformation of the network.

Keywords: associativity, productive network, SMEs, footwear sector, Ecuador.

JEL: M1 Business Administration; D7 Analysis of collective decision-making.

\section{Resumo}

Esta pesquisa apresenta um caso aplicado a pequenas e médias empresas do setor calçadista da província de Tungurahua. O objetivo é determinar a viabilidade ou cooperação instrumental mais viável para a formação de uma rede produtiva. Os empresários são questionados sobre os fatores críticos, sua importância e graus de possíveis complementos para identificar os aspectos que motivam a conformação da rede que se refletem nos índices de cooperação benéfica (ICP). Por fim, estabelece-se que o ICP mais alto corresponda ao fator de produção, razão pela qual se recomenda iniciar ações para a conformação da rede.

Palavras-chave: asociatividad, produtiva vermelha, pequeñas e medianas empresas, setor calzado, Equador.

JEL: M1 Administração de Empresas; Análise D7 de tomada de decisão coletiva. 


\section{Introducción}

L

a provincia de Tungurahua está ubicada en la región Sierra en el centro del Ecuador, con una superficie aproximada de $3.400 \mathrm{~km}^{2}$. La población de la provincia, de acuerdo con el Censo de Población y Vivienda del Instituto Nacional de Estadística y Censos (2010), es de 504.683 habitantes, con una densidad poblacional de 150 habitantes por $\mathrm{km}^{2}$. El $43 \%$ de la población se encuentra en la zona rural, mientras que el $57 \%$ está en la zona urbana. La capital de la provincia es Ambato, que concentra una población de 326.688 habitantes y 23.869 establecimientos económicos (Ministerio Coordinador de Producción, Empleo y Competitividad 2011).

La Agenda Tungurahua 2015-2017 describe a la provincia considerando a la población económicamente activa (PEA) como principalmente agrícola con el $26,94 \%$ PEA, manufacturera con el $18,63 \%$, comercial con el 16,55\%, y de servicios varios con el $14,22 \%$. Dentro de sus objetivos provinciales se encuentra el incremento del ingreso, para cuyo efecto se han definido políticas de fomento productivo que, a través de la Estrategia Agropecuaria, la Agenda de Turismo, la Agenda de Competitividad y la Agenda Artesanal, han direccionado las inversiones del sector público y privado (Gobierno Provincial de Tungurahua 2015).

En la provincia de Tungurahua, en el año 2014, se decidió intensificar la matriz productiva, en virtud de sus fortalezas en los ámbitos productivo, agropecuario, comercial e industrial. La generación de ingresos y de fuentes de trabajo tienen como base la transformación y creación de nuevos productos, así como la sustitución de importaciones y maximización de exportaciones.

Lo indicado anteriormente hace notar la gran importancia de transitar, desde una posición de productores de materias primas a productores de bienes terminados creando valor agregado y optimizando recursos, mediante de una buena gestión de los recursos intangibles y tangibles, como en el caso de 
la gestión del conocimiento y la asociatividad; de esta manera, se propenderá a la mejora de la producción y la productividad de las empresas localizadas en la provincia de Tungurahua.

La acción del Gobierno Provincial de Tungurahua ha girado sobre grandes objetivos trazados en la Agenda de Tungurahua, desde el año 2003: agua, gente y trabajo, que, bajo principios de representatividad, corresponsabilidad y responsabilidad, ha consolidado un modelo de gestión que permite promover el desarrollo de la provincia. La Agenda para la Transformación Productiva Territorial de Tungurahua manifiesta que las pyme tienen una importancia estratégica en el crecimiento de la economía para el desarrollo del aparato productivo local, y la mejor posición competitiva del país (Ministerio Coordinador de Producción, Empleo y Competitividad 2011).

Cabe indicar que las pyme, según la clasificación por determinantes de la legislación ecuatoriana, comprenden a las micro, pequeñas y medianas empresas. Las medianas tipo A tienen entre 100 y 199 empleados, las medianas tipo B tienen entre 50 y 99 empleados, las pequeñas, de 10 a 49 empleados, y las micro tienen menos de 10 empleados (INEC 2012). De este conglomerado de empresas, las pequeñas y las medianas son las que presentan mejores condiciones para asociarse, gestionar los recursos y proyectarse al futuro.

La provincia de Tungurahua ha tenido tradicionalmente una presencia muy fuerte en la producción de calzado; resulta interesante mencionar que a pesar de que esta provincia representa, aproximadamente, el 1,2\% de la extensión del territorio nacional $\left(3.400 \mathrm{~km}^{2}\right.$ de $\left.283.560 \mathrm{~km}^{2}\right)$, produce alrededor del 50\% de la producción nacional de calzado (La Hora 2016).

El Gobierno Provincial de Tungurahua, con el propósito de dar cumplimiento al objetivo estratégico de estimular la actividad económica local con la participación de actores públicos y privados, propicia un clima de negocios para el mejoramiento de la productividad y la creación de empleo, y propone elaborar un proyecto bajo el eslogan "Un pueblo, un producto" (Gobierno Provincial de Tungurahua 2015), para asociar a los productores de calzado a fin de elevar la competitividad del sector mediante el mejoramiento de los sistemas de producción.

El sector del calzado de la provincia, en los últimos años, de acuerdo con el Ministerio de Industrias y Productividad (2011), ha tenido un importante desarrollo; así en el año 2008 la venta interna de calzado nacional fue de US 
\$ 165,7 millones, mientras que en el año 2011 ascendió a US \$ 318 millones. En el mismo sentido, la Cámara Nacional de Calzado (CALTU) señaló que en el año 2008 la producción de calzado fue de 15 millones de pares de zapatos, mientras que en 2011 se produjeron 28,8 millones de pares de zapatos (El Comercio 2016).

Como se puede apreciar, el crecimiento del sector del calzado en la provincia de Tungurahua, en tres años, es evidente con un incremento en su producción y ventas de alrededor del $92 \%$, lo que se explica por las políticas arancelarias del gobierno ecuatoriano implementadas en esa época, consistentes en un gravamen de US \$ 6.00 más el $10 \%$ ad valorem a cada par de zapatos importado, con lo que se consiguió dinamizar la producción y ventas de calzado al interior del país.

Los directivos de CALTU manifestaron que el sector del calzado, hasta 2013, creció en un 80\%; mientras que, del año 2014 a 2015, hubo un decrecimiento del $30 \%$, por cuyo efecto cerraron 187 unidades productivas en 2015; del mismo modo y en la misma fuente se da a conocer que la disminución de ventas y el incremento de la cartera vencida, en el primer trimestre de 2016, dio lugar al cierre de nuevas unidades de producción de calzado (La Hora 2016). Como se observa, la situación descrita es alarmante, de tal modo que exige tomar medidas internas que permitan que este sector salga a flote: una de ellas es optar por la búsqueda de mecanismos de asociatividad que permitan la creación de redes productivas.

Las pyme enfrentan retos que les exigen adaptación, mejoramiento de sus estructuras, procesos y modelos de gestión, a fin de ser más competitivas. Como fue conocido en años anteriores, el sector del calzado mostró un crecimiento notable debido a políticas favorables de gobierno, sin embargo, a partir del año 2016, se detectó un impresionante decrecimiento en razón de la política económica que adoptó el Ecuador, causando una afección significativa y, por tal razón, surge la necesidad de asociarse con el propósito de potenciar fortalezas, superar debilidades y aprovechar las oportunidades del entorno.

Bajo esta perspectiva, la presente investigación propone determinar los aspectos que motivan la conformación de una red productiva dentro del sector del calzado. 
La asociatividad, fundamentada en la generación de aptitudes relacionales en la que los participantes comparten conocimientos técnicos y logran mejorar su situación de trabajo, es una estrategia utilizada por los diferentes sectores de la sociedad civil (Maldovan y Dzembrowski 2009); es, por tanto, una perspectiva relevante y oportuna, en virtud de que es posible llegar a unir esfuerzos individuales para propósitos colectivos. Con esta finalidad, además, es valorable contar con la apertura de los empresarios para tomar acciones participativas previas a la formación de la red, a partir de lo cual se puede indagar, desde los actores, elementos de juicio que contribuyan a la promoción de redes productivas formadas por las pyme del sector del calzado y conseguir el apalancamiento necesario para que el sector pueda salir de la crisis.

En la presente investigación se determina la factibilidad instrumental de la conformación de una red productiva de pyme del sector del calzado, así como establecer las posibilidades que existentes de implementar actividades concretas de complementación y cooperación entre las pyme del sector. ${ }^{1}$

\section{La pequeña y mediana empresa}

En la historia de la humanidad se ha observado que los escenarios son cambiantes y que en épocas de escasez o de grandes dificultades se evidencia un desarrollo mayor que en épocas de bonanza; es así que, en algunas ocasiones, a partir de las guerras se han generado inventos y procesos innovadores, surgiendo nuevas maneras de hacer las cosas que conlleven mejores maneras de vivir.

La presencia de las pyme en América Latina tiene un alto impacto en la economía de la región, y es una fuente importante de ingresos y empleo. En América Latina las pyme generan el alrededor del 60\% de empleo y el $30 \%$ del PIB, mientras que en los países de la OCDE generan alrededor del $60 \%$ del PIB y entre 50 y $60 \%$ de empleo (Naciones Unidas, CEPAL y OCDE 2012).

1. Esta investigación no se encarga de evaluar la factibilidad económica dado que se lo considera que es objeto de una nueva investigación. 
Sin embargo, se debe mencionar que los niveles salariales y de productividad de las pyme no son satisfactorios pues enfrentan una gran cantidad de dificultades que deben ser investigadas en contexto (OIT 2015) a fin de contribuir a la formulación de políticas que busquen el crecimiento sostenido en los diversos vectores que comprenden su heterogeneidad.

La Fundación para el Desarrollo Sostenible es una organización internacional que promueve el desarrollo competitivo de la pyme en América Latina desde 1984, a través de su fortalecimiento, y sostiene que aproximadamente el 97\% de la población empresarial corresponde a este sector. Por otro lado, solamente el 30\% del PIB proviene de las pyme, frente al 70\% proveniente de grandes empresas (Naciones Unidas, CEPAL y OCDE 2012), con lo que se puede apreciar que aunque se trate de un gran número de pyme, su aporte al PIB es reducido.

En América Latina las pyme enfrentan problemas relativos a la situación sociopolítica que les ha tocado vivir durante distintos gobiernos, como lo relata Pérez Fernández (2015); así, durante los 70 se caracterizaron por dictaduras, seguidas por la socialdemocracia vivida en la década del 80; mientras que en los 90 hubo una marcada tendencia hacia la derecha. Al iniciar el siglo XXI, emergió una nueva izquierda que generó muchas expectativas (Paz y Miño 2015); finalmente, en la segunda década del siglo XXI, aparecen serias posibilidades de cambio de dirección de la situación sociopolítica en América Latina.

Es en este escenario que las pyme, han tenido un apoyo limitado para su desarrollo, más aún, a pesar de que en años anteriores hubo determinadas políticas de incentivos, que hoy en día están atravesando dificultades, por su pérdida de competitividad, y baja productividad de las pyme que se encuentran entre el 25 y el $50 \%$ de las grandes empresas, razón por la cual se deben buscar alternativas para su desarrollo (Vergara 2005).

En Ecuador, en un estudio realizado por Jácome y King (2013), se expresa la importancia de las pyme en el tejido empresarial ecuatoriano, de tal manera que inclusive el MIPRO extendió una invitación a inversionistas, académicos, emprendedores y sector productivo en general a tomar acciones para mejorar la situación de las pyme en el desarrollo del país. 


\section{Asociatividad}

A medida que la globalización avanza, la tecnología se ha intensificado, los mercados se han globalizado y se ha incrementado la volatilidad de los entornos competitivos, ha sido imprescindible que las organizaciones acudan a buscar acuerdos de cooperación. Actualmente, es muy difundido el hecho de que los escenarios son muy competitivos y que las empresas se esfuerzan por conseguir una buena posición en el mercado en donde las acciones de cooperación cobran mayor importancia (Rodríguez 2014). Los acuerdos de cooperación pueden tener distintas formas según las condiciones, requerimientos y recursos particulares de cada situación; así, se pueden formar alianzas estratégicas, consorcios y joint ventures, entre otros, con lo que se construyen redes organizativas (Galán y Castro 2014) que buscan conseguir ventajas competitivas sostenibles.

Dependiendo de la región del mundo donde se aborde el tema de la asociatividad, como campo de estudio, existen diferentes consideraciones respecto al modo de configurar las relaciones entre diferentes empresas del mismo sector o de sectores complementarios. La manera más común de teorizar estas relaciones es por medio de cadenas productivas, redes productivas y cluster.

En la mayoría de los casos las diferencias encontradas entre la teoría referente a la asociatividad son pequeñas y están más orientadas por la tradición desarrollada en cada país (McCormick y Morales 2006).

Pallares Villegas (2003) dice que la epistemología del término asociatividad ha evolucionado, y alrededor de 2001 la tendencia ha sido utilizar la acepción de asociaciones; esto se complementa con algunas orientaciones que conciben la asociatividad empresarial como una respuesta de los pequeños productores a la internacionalización de las economías.

La asociatividad es una alternativa para el desarrollo y crecimiento de las pyme (Liendo y Martínez 2001), y hay que desarrollar aspectos conceptuales de cooperación, así como las dimensiones con las que se debe trabajar (Maldovan y Dzenbrowski 2009).

La asociatividad empresarial es un concepto que ha sido objeto de mucho interés en la teoría económica, especialmente a partir del trabajo de Michael Porter (1998) y su diamante de competitividad que, como se conoce, ha sido 
ampliamente aplicado en la práctica por importantes sectores empresariales de América Latina (Jácome y King 2013).

Cuando se acude a la asociatividad, las empresas se ponen en marcha de diversas maneras, en unas ocasiones siguiendo una secuencia vertical en cuyo caso se trata de cadenas mediante las cuales se generan o consumen bienes o servicios; y en otras ocasiones se asocian mediante redes. Una red es el conjunto de personas o empresas de la misma naturaleza que hacen acuerdos para obtener beneficios comunes alrededor a un proyecto específico; si la red es de tipo productivo se trata de una red productiva, en la cual tiene lugar la transformación de insumos en productos.

Las cadenas productivas no caben en la definición de cluster debido a que tienen débiles relaciones con los territorios y por ende con las instituciones competentes en ofrecer apoyo a los distintos sectores productivos, por el mismo motivo, sus relaciones con el Estado no son claras y los esfuerzos por gestionar apoyo estatal son poco eficaces (McCormick y Morales 2006).

En cambio, un cluster es un grupo de empresas o instituciones interconectadas, asociadas y ligadas por actividades e intereses comunes y complementarios, geográficamente próximos y pertenecientes al mismo sector industrial (Valdivia Altamirano 2011). Por lo que, a través de la variedad de relaciones que se van estableciendo, generan ventajas para cada uno de los particiantes (Hendry y otros 2014).

Según Navarro Arancegui (2003), al vocablo clusters se le ha dado diferentes significados dependiendo de las corrientes que siguen los autores, dejando en claro que se trata de diferentes ocpciones en torno a un aspecto en especial, sin dejar de lado a las organizaciones que lo integran y el tipo de relaciones ente ellas. El estudio de clusters oscila alrededor de la polémica de su extensión geográfica, quiénes lo conforman, cómo es la relación de quiénes lo conforman, el flujo de materias primas, el flujo de conocimiento que los enlaza, su nivel de conciencia colectiva y su nivel de compromiso para el desarrollo del cluster por parte de quienes lo integran (Porter 1998).

En cuanto a las redes, en la práctica, se pueden encontrar dos tipos de redes: formales e informales, de acuerdo con la manera en que funcionen o estén constituidas; sin embargo, las redes formales tienen la ventaja de facilitar el acceso al know how, lo que contribuye a la creación y aplicación de nuevos 
conocimientos que, a su vez, conllevan al incremento de la competitividad; por lo tanto, es conveniente promover la construcción de redes formales.

Cabe reiterar que la creación de redes productivas en las pyme aparece como un mecanismo oportuno para enfrentar los problemas emergentes, pues el contexto económico en que estas se desenvuelven presenta cada vez mayores requerimientos a nivel nacional e internacional, por lo que contantemente deben mejorar su competitividad.

Para la formación de una red productiva es menester buscar oportunidades de cooperación (Marchetti 2008); oportunidades que deberían ser exploradas desde distintos frentes hasta encontrar un punto de convergencia para emprender esfuerzos de trabajo conjunto. Si bien toda empresa tiene debilidades y fortalezas, se debe identificar las fuerzas impulsoras para la formación de una red tomando en cuenta los aspectos imprescindibles dentro de su actividad productiva como son los procesos de producción, el mercado, la tecnología, las finanzas, el abastecimiento y otros que dependerán básicamente de la naturaleza de la pyme.

Las ventajas competitivas generadas por trabajar en forma asociativa, sea en redes, cadenas o cluster, propician la interacción conjunta de los esfuerzos individuales de los agentes asociados, cuyo resultado es superior a la suma de sus partes (Raines 2000). El efecto sinérgico que puede obtenerse de la asociación de empresarios dotados de un alto nivel de compromiso puede dar un giro positivo a un sector productivo; por tanto, resulta primordial buscar la participación de un grupo de empresarios que compartan la visión de formación de una red productiva para beneficio mutuo y con quienes se debe trabajar desde un inicio para la elaboración del proyecto de planificación y consolidación de la red.

En la formación de cadenas productivas, se considera que, además de determinar factores críticos de éxito, es importante identificar los intereses comunes entre los actores, los cuellos de botella en sus relaciones y las maneras de superarlos (CONCOPE 2011). Así mismo, en el caso de las redes productivas, se recomienda además que se busque identificar ventajas en los productos, tecnología, habilidades o recursos de las empresas para que sirvan de apoyo mutuo y puntal de la red. De modo similar, en cuanto a las debilidades, es necesario identificarlas a fin de que las empresas puedan disminuir o mitigarlas mediante la complementación. 
Marchetti (2008) hace algunas puntualizaciones para la formación de redes productivas; así, manifiesta que es menester explorar los distintos factores de cooperación, entre los que menciona al abastecimiento, producto, mercado, tecnologías, logística, entre otros, dando prioridad a uno de ellos de acuerdo a su relevancia comparativa entre las pyme de potencial asociación. Por otro lado, recomienda tomar en cuenta a los aspectos involucrados dentro de cada uno de ellos.

En la búsqueda de posibles aliados lo más importante es determinar la voluntad para asociarse y la predisposición para hacer acuerdos, sin lo cual aunque se contara con muchas fortalezas, no sería posible iniciar la conformación de una red.

\section{Proceso para la conformación de una red productiva}

Para la conformación de un grupo de personas orientado a la ejecución del cumplimiento de objetivos comunes, es necesario determinar la factibilidad instrumental. Esta es una factibilidad administrativa, a través de la cual se pone de manifiesto el poder generado por las voluntades y acuerdos de las personas (Dussel 2006). En el caso de la conformación de una red productiva se aplica la misma idea, pues mediante la determinación de la factibilidad instrumental se busca determinar las cooperaciones que resulten provechosas para el efecto.

Marchetti (2008) explica el proceso para la conformación de una red, e indica que es primordial identificar los factores críticos de cooperación que constituyen aquellos aspectos de la cadena de valor que de acuerdo a la misión de la empresa o sector económico pueden tener mayor o menor importancia a criterio de los propios actores. Cada factor crítico cuenta con diversas fuentes de relación que son las oportunidades de cooperar. Cada oportunidad tiene su propio nivel de importancia, de acuerdo con la unanimidad de criterios del grupo de empresarios.

Es imprescindible acudir a la investigación de campo para determinar los grados de posibles complementaciones entre las empresas, para tener una clara perspectiva de cuán dispuesto está un empresario a asociarse para un 
propósito determinado. Esto constituye la probabilidad de interactuar y compartir que tienen las empresas dentro de cada factor de posible cooperación.

Al disponer de los datos correspondientes de los factores críticos, su importancia y las posibles complementaciones, se puede determinar la tasa de cooperación ponderada, que es la proporción de cada posible complementación dentro de cada factor crítico.

Finalmente, al haber conseguido la información señalada anteriormente, se cuenta con los insumos necesarios para el cálculo de un índice de cooperación provechosa (ICP), que representa la proporción total de la tasa de cooperación ponderada de cada factor crítico con respecto a su propia ponderación. La determinación de estos valores permite establecer la factibilidad instrumental de formación de una red partiendo de las relaciones más significativas que sirvan de base para la toma de decisiones relativas a la asociatividad.

Un punto neural en la conformación de una red es la búsqueda y hallazgo de posibles aliados porque son relaciones basadas en el compromiso y confianza mutua, de tal manera que al inicio de la red se considera importante la evaluación de las relaciones. Por otro lado, es imprescindible evaluar también las capacidades de los posibles aliados y el estado actual de cada empresa por cuando se trata de avanzar de modo sinérgico como red en todos los frentes que se propongan y no dedicarse a solventar situaciones negativas preexistentes.

En el proceso de conformación de la red aparecerán confrontaciones y conflictos, pues el ser humano, y más aún el empresario, está preparado para competir y no para cooperar, es por naturaleza individualista, presto para defender los intereses personales antes que los colectivos; por esta razón, la metodología para la conformación de redes recomienda que se opere a base de un proyecto que plasme los propósitos, acciones, actores y compromisos de los asociados. Este proyecto debe tener un facilitador que propicie el entendimiento entre los participantes y guíe las acciones hacia la cooperación.

\section{Metodología}

La presente investigación tiene un enfoque cualitativo y es de tipo descriptivo. Las unidades de análisis son las pyme de la provincia de Tungurahua. Tomando en cuenta la cantidad de unidades de análisis, las variables y 
la temporalidad, esta investigación utiliza un diseño intensivo, multivariado y transversal (Ynoub 2015).

El sector del calzado en la provincia de Tungurahua está compuesto por 642 microempresas, 71 empresas pequeñas, 14 empresas medianas y 4 empresas grandes (INEC 2014). Por lo indicado, el universo del presente estudio está constituido por 85 pyme de calzado.

En una primera etapa se realizó un grupo focal con cinco expertos, quienes fueron elegidos considerando a su participación en el sector productivo, su nivel de interés en el tema de asociatividad y su predisposición para colaborar en la presente investigación. Con la información obtenida se identificaron los factores críticos, fuentes de relación, posibles complementaciones y ventajas de la potencial conformación de una red. Esta información permitió determinar los aspectos que motivan a asociarse (tabla 1). Los insumos informativos constituyeron la base para elaborar la herramienta que consiste en un cuestionario compuesto por 45 ítems.

El cuestionario fue validado por un grupo de expertos y está estructurado de la siguiente manera: una pregunta de modo general indaga la predisposición para asociarse, una pregunta para cada uno de los cuatro factores: abastecimiento, producción, mercado y finanzas en cuanto al peso o ponderación dentro del quehacer empresarial, cinco preguntas dentro de cada factor con respecto a la importancia de esa oportunidad de cooperación, $y$, cinco preguntas referentes a la probabilidad de la complementación efectiva en cada oportunidad de cooperación.

El cuestionario se aplicó al universo ( 85 pyme) mediante encuesta y se consiguió una tasa de respuesta del $25 \%$ que corresponde a 21 pyme. Cabe mencionar que si se hubiera aplicado un muestreo aleatorio simple, los parámetros estadísticos para conseguir el mismo tamaño de muestra habrían correspondido al $90 \%$ de confianza y $16 \%$ de error. Sin embargo, no se consideró apropiado utilizar el muestreo aleatorio simple, porque, al tratarse de un tema de asociatividad, la voluntad de los empresarios es un factor determinante y de suprema importancia, a partir de la cual se toman decisiones.

El procedimiento utilizado tiene como base la metodología para la creación de redes propuesta por Marchetti (2008). El tratamiento de los datos se llevó a cabo utilizando el software MS Excel. 
Una vez tabulados los datos se determinó el peso de los factores críticos de cooperación que indica su importancia relativa en la cadena de valor. Además, se determinó el peso de las posibles cooperaciones dentro del factor crítico, que indican la importancia que los empresarios le han dado a ese aspecto. Luego, se estableció la probabilidad de que en la práctica ocurra cada oportunidad de cooperación, que en la tabla 2 figura como grado de posible complementación (la suma de los pesos asignados a los factores, así como de las posibles cooperaciones dentro de cada factor, debe sumar ciento por ciento, no así la probabilidad de complementación). Los porcentajes reportados se basaron en los criterios proporcionados por los empresarios en los diferentes aspectos.

En la tercera columna de la tabla 2 se encuentran los valores de la tasa de cooperación ponderada que se estableció a través del producto de los pesos de las posibles cooperaciones y el grado de las posibles complementaciones y se refiere a la proporción de cada posible complementación dentro de cada factor crítico, como se explicó en la parte correspondiente al proceso para la conformación de una red.

Con los datos conseguidos hasta este punto se procedió al cálculo del índice de cooperación provechosa (ICP) en cada uno de los factores críticos de cooperación: abastecimiento, producción, mercado y finanzas, con cada una de las cinco posibles cooperaciones.

El ICP indica la factibilidad instrumental, es decir, el resultado de relacionar las ponderaciones de cada una de las posibles complementaciones y su probabilidad de complementación en cada factor crítico; se calculó multiplicando el peso de cada factor crítico por el total de la tasa de cooperación ponderada correspondiente. El ICP representa la cooperación provechosa en cada factor crítico. Finalmente, se identificó el valor más alto del ICP que sirve para seleccionar el factor crítico recomendable para empezar esfuerzos de cooperación.

\section{Resultados}

El 90\% de empresarios manifiestan interés para asociarse, lo cual proporciona un escenario favorable en el que existen buenas perspectivas para la formación de redes productivas. 
Los resultados de la entrevista a los cinco expertos que se muestran en la tabla 1 están desagregados en factores y fuentes de relación, posibles complementaciones y posibles ventajas que se obtendrían al asociarse.

Tabla 1

\section{Criterios para la formación de la red productiva de las pyme de calzado}

\begin{tabular}{|c|c|c|}
\hline $\begin{array}{l}\text { Factor/fuente } \\
\text { de relación }\end{array}$ & Posible complementación & Posible ventaja \\
\hline \multicolumn{3}{|c|}{ Abastecimiento } \\
\hline $\begin{array}{l}\text { Método de selección } \\
\text { de proveedores } \\
\text { compartido. }\end{array}$ & $\begin{array}{l}\text { Compartir un método para } \\
\text { la selección de proveedores. }\end{array}$ & $\begin{array}{l}\text { Mejor el servicio y la calidad } \\
\text { de la materia prima. }\end{array}$ \\
\hline Proveedores comunes. & Compartir proveedores. & Conseguir mejores precios. \\
\hline $\begin{array}{l}\text { Gestión de compras } \\
\text { conjuntas. }\end{array}$ & Hacer compras conjuntas. & Mejorar el poder de negociación. \\
\hline Transporte compartido. & $\begin{array}{l}\text { Compartir el transporte } \\
\text { en la adquisición de materia. }\end{array}$ & Disminuir costos de transportación. \\
\hline $\begin{array}{l}\text { Ubicación común } \\
\text { de materias primas. }\end{array}$ & $\begin{array}{l}\text { Tener bodegas compartidas } \\
\text { para almacenar productos. }\end{array}$ & Reducir costos de almacenamiento. \\
\hline \multicolumn{3}{|c|}{ Producción } \\
\hline $\begin{array}{l}\text { Métodos y procesos de } \\
\text { producción compartidos. }\end{array}$ & $\begin{array}{l}\text { Compartir métodos } \\
\text { y procesos de producción. }\end{array}$ & Estandarización de procesos. \\
\hline Fabricación compartida. & $\begin{array}{l}\text { Fabricar partes o piezas } \\
\text { en forma compartida. }\end{array}$ & Optimización de recursos. \\
\hline $\begin{array}{l}\text { Instalaciones } \\
\text { compartidas. }\end{array}$ & $\begin{array}{l}\text { Compartir instalaciones } \\
\text { para la producción. }\end{array}$ & Reducción de costos. \\
\hline $\begin{array}{l}\text { Control de calidad } \\
\text { conjunto. }\end{array}$ & $\begin{array}{l}\text { Hacer control de calidad } \\
\text { en forma compartida. }\end{array}$ & Estandarización de la calidad. \\
\hline $\begin{array}{l}\text { Apoyo mutuo } \\
\text { en mantenimiento } \\
\text { de planta. }\end{array}$ & $\begin{array}{l}\text { Apoyarse en el mantenimiento } \\
\text { de planta y equipos. }\end{array}$ & $\begin{array}{l}\text { Disminución de costos } \\
\text { de mantenimiento. }\end{array}$ \\
\hline \multicolumn{3}{|c|}{ Mercado } \\
\hline $\begin{array}{l}\text { Investigación } \\
\text { de mercados conjunta. }\end{array}$ & $\begin{array}{l}\text { Abrir mercados y planear } \\
\text { ventas conjuntas. }\end{array}$ & $\begin{array}{l}\text { Reducción de costos de búsqueda } \\
\text { de clientes. }\end{array}$ \\
\hline
\end{tabular}




\begin{tabular}{|l|l|l|}
\hline $\begin{array}{l}\text { Mercado geográfico } \\
\text { común. }\end{array}$ & $\begin{array}{l}\text { Hacer campañas publicitarias } \\
\text { conjuntas. }\end{array}$ & $\begin{array}{l}\text { Reforzar imagen y disminución } \\
\text { de costos. }\end{array}$ \\
\hline Canales comunes. & $\begin{array}{l}\text { Distribuir físicamente } \\
\text { de manera compartida. }\end{array}$ & $\begin{array}{l}\text { Mejoramiento de la logística } \\
\text { y reducción de costos. }\end{array}$ \\
\hline Consumidores comunes. & Compartir fuerza de ventas. & $\begin{array}{l}\text { Mejor cobertura de mercado } \\
\text { y reducción de costos. }\end{array}$ \\
\hline Marcas compartidas. & $\begin{array}{l}\text { Compartir o complementar } \\
\text { marcas. }\end{array}$ & Sinergia de marcas. \\
\hline \multicolumn{2}{|c|}{ Finanzas } \\
\hline $\begin{array}{l}\text { Búsqueda conjunta } \\
\text { de fuentes } \\
\text { de financiamiento. }\end{array}$ & $\begin{array}{l}\text { Buscar fuentes } \\
\text { de financiamiento apropiadas. }\end{array}$ & $\begin{array}{l}\text { Ampliar las opciones } \\
\text { de financiamiento. }\end{array}$ \\
\hline $\begin{array}{l}\text { Necesidades similares } \\
\text { de financiamiento. }\end{array}$ & $\begin{array}{l}\text { Negociar conjuntamente } \\
\text { el financiamiento. }\end{array}$ & $\begin{array}{l}\text { Mejorar los términos } \\
\text { de negociación. }\end{array}$ \\
\hline $\begin{array}{l}\text { Capital de trabajo } \\
\text { compartido. }\end{array}$ & $\begin{array}{l}\text { Formar un capital común } \\
\text { con el aporte de todos. }\end{array}$ & $\begin{array}{l}\text { Incrementar el capital } \\
\text { y propiciar mayores réditos. }\end{array}$ \\
\hline Reinversión. & $\begin{array}{l}\text { Hacer nuevas inversiones } \\
\text { con los excedentes. }\end{array}$ & Generar nuevos beneficios. \\
\hline $\begin{array}{l}\text { Manejo financiero } \\
\text { conjunto. }\end{array}$ & $\begin{array}{l}\text { Tener un solo manejo } \\
\text { de las finanzas. }\end{array}$ & $\begin{array}{l}\text { Incrementar la eficiencia } \\
\text { en el manejo de las finanzas. }\end{array}$ \\
\hline
\end{tabular}

Fuente y elaboración: autores.

Las fuentes de relación entre los empresarios gozan de amplitud de opciones para su complementación con lo que se puede generar ventajas tanto de manera individual como para el sector. Estas opciones de complementación se resumen en cuatro puntos: a) compartir aspectos fiscos como infraestructura o equipos; b) compartir intangibles como marcas, métodos o información; c) compartir personas como proveedores o vendedores; y d) compartir acciones como fabricar de manera conjunta, hacer control de calidad, abrir mercados o hacer negociaciones.

A partir de la tabla 1, se estructuró el cuestionario aplicado a los empresarios cuyo contenido se resume en la primera columna de la tabla 2. La importancia de cada factor, cooperaciones y grados de posible complementación se cuantificaron a partir de la tabulación de las respuestas del cuestionario, los resultados están en la tabla 2. 


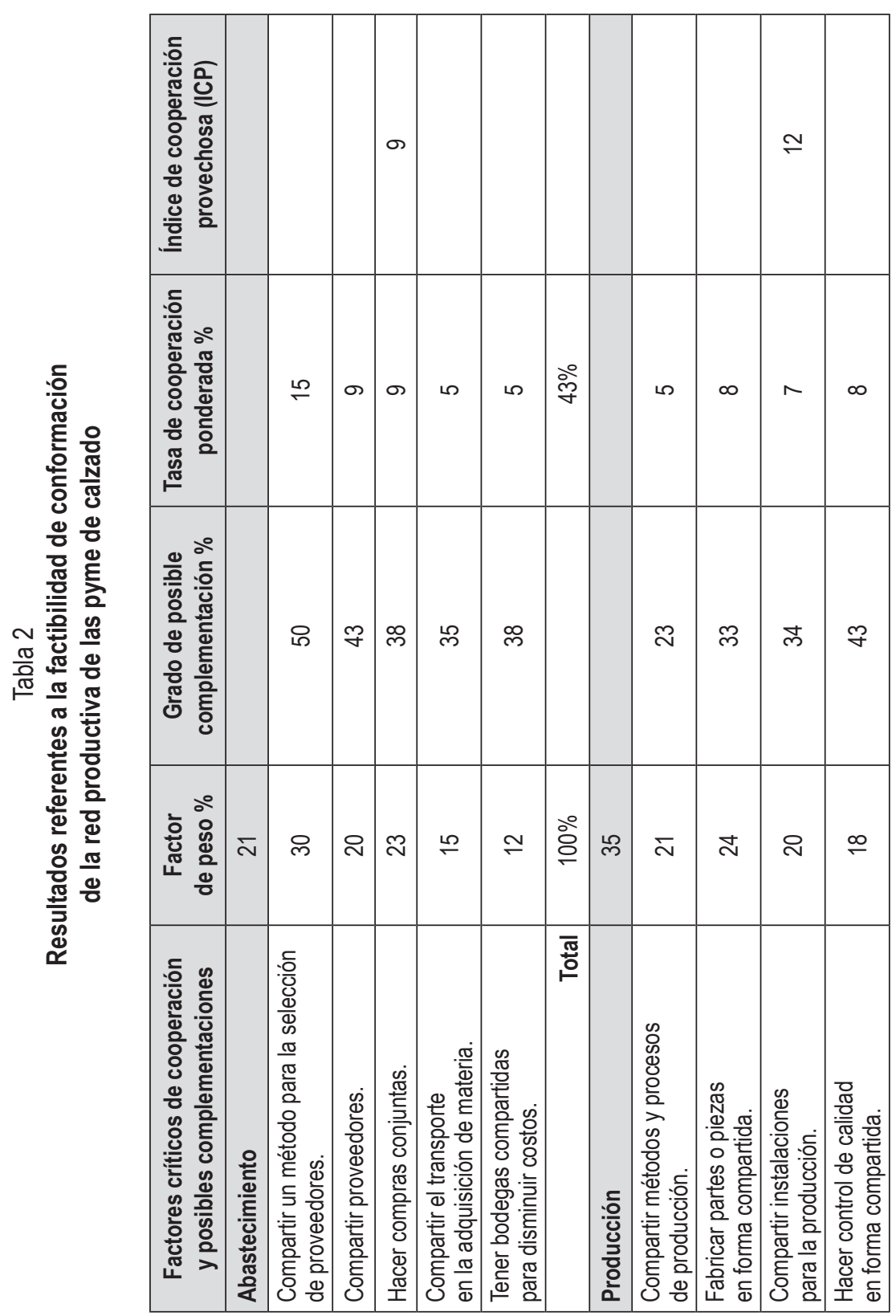




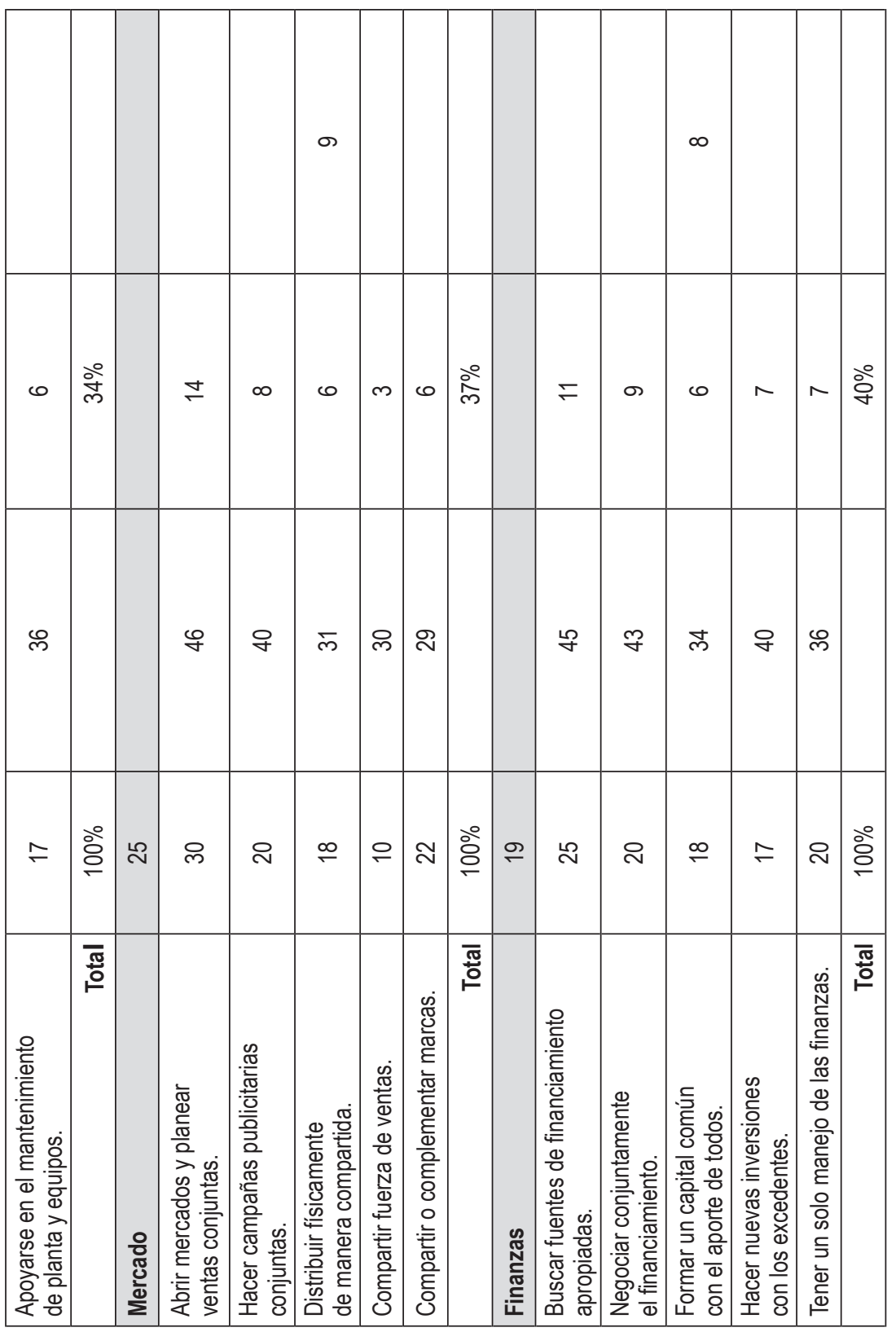

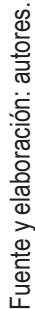


El rango de variación del peso o importancia de cada factor crítico es del 16\% (diferencia entre 19 y $35 \%$ ), encontrándose en el extremo superior a producción y en el inferior al de finanzas, mientras que en los factores de abastecimiento y mercado se reportan cifras relativamente similares $(21 \mathrm{y}$ $25 \%$ ), como se aprecia, ninguno de los factores presenta valores extremadamente altos o bajos.

La posible complementación de mayor importancia dentro del factor abastecimiento corresponde a compartir un método de selección de proveedores, mientras que dentro del factor producción se refiere a producir piezas en forma compartida. De modo análogo, en el factor mercado la complementación de mayor importancia corresponde a abrir mercados y planear ventas conjuntas, mientras que para el factor finanzas el valor más alto es para buscar fuentes de financiamiento apropiadas. Al observar la tabla 2 se aprecia que los valores de la importancia de las posibles complementaciones en cada factor crítico son altos, medios y bajos.

Cabe mencionar que los valores de las ponderaciones que denotan la importancia de las posibles complementaciones se ven afectados por el grado de las posibles complementaciones que representa su probabilidad de ocurrencia. Se puede apreciar de modo general que estas probabilidades oscilan entre el 30 y el 50\%, con lo que establecen perspectivas medio-bajas de su implementación u ocurrencia.

Los resultados conseguidos hasta el momento direccionan los cálculos posteriores que corresponden a la tasa de cooperación ponderada e ICP, cuyos valores se aprecian en la cuarta y quinta columna de la tabla 2.

El total más alto de la tasa de cooperación ponderada es para el factor abastecimiento, seguido por mercado, finanzas y producción (43, 40, 37 y $34 \%$ respectivamente). Es relevante apreciar que en los tres primeros casos el total se ve influenciado por una sola de las posible complementaciones que exhibe un valor notoriamente más alto que el resto $(15,14$ y $11 \%$ en forma respectiva).

En el caso del factor de producción, las diferencias son ínfimas, lo que permite establecer que las posibles complementaciones del factor de producción tengan probabilidades relativamente similares, hecho que es conveniente para los propósitos del funcionamiento de la red productiva, dado que las 
cinco posibles complementaciones tienen posibilidades de implementación efectiva.

Finalmente, se reporta que el ICP más alto tiene un valor de 12, lo que sugiere que la red productiva de las pyme del sector del calzado debe iniciar por unir esfuerzos en los aspectos relacionados a ese factor en base a los cuales se debe tomar decisiones para hacer los acuerdos pertinentes al menos a corto plazo.

Se determina, entonces, que este ICP de 12 corresponde al factor producción con sus posibles cooperaciones: compartir métodos y procesos de producción, fabricar piezas en forma compartida, compartir instalaciones para producción, hacer control de calidad en forma compartida y apoyarse en el mantenimiento de planta y equipos.

\section{Conclusiones}

De la literatura revisada se desprende que las pyme tienen varios problemas que deben ser solucionados, pues son importantes en virtud de que constituyen un gran porcentaje del tejido empresarial de los países de América Latina.

De la entrevista a los empresarios se concluye que las pyme en la provincia de Tungurahua aún no han dado pasos firmes para formar asociaciones productivas formalmente establecidas con un funcionamiento que les permita alcanzar un desarrollo sostenible, incrementar su mercado y elevar su nivel de competitividad. Sin embargo, resulta alentador identificar que los empresarios del sector del calzado presentan interés para la formación de una red productiva que de ser bien administrada, mejoraría las perspectivas del sector.

La importancia asignada a cada una de las posibles complementaciones: compartir un método de selección de proveedores, producir piezas en forma compartida, abrir mercados y buscar fuentes de financiamiento apropiadas, es la más alta, si se compara con las cuatro restantes, dentro de cada factor, de lo que se concluye que existen varias oportunidades para una potencial conformación de redes productivas desde diferentes frentes. 
Se recomienda que el factor producción sea el punto de partida para la conformación de la red y deberían detallar las actividades al interior de cada una de las posibles complementaciones con el fin de planificar su efectiva implementación para la conformación de la red.

También se recomienda iniciar la red a partir de factor producción, que resulta de la interacción entre el grado de posible complementación y la tasa de cooperación ponderada.

Las posibles cooperaciones relacionadas con el factor producción son las que ofrecerán mayores fortalezas, pudiendo considerar que se encuentran en estado de madurez.

Para el buen funcionamiento de la red productiva no es suficiente contar con buenos procesos, recursos técnicos y materias primas bien seleccionadas, sino que es imperativo destacar la necesidad de que los empresarios estén dispuestos a abrir su mentalidad y espíritu hacia la solidaridad, la transparencia y la honestidad, que son valores condicionantes que deben ser cultivados y consolidados.

Para que los buenos resultados conseguidos del trabajo en red sean conservados y mejorados en el tiempo, es necesario que la factibilidad instrumental se mantenga vigente, para reflejar el poder de ejecución proveniente de las voluntades y acuerdos entre las personas.

Los empresarios participantes deberán poner sobre la mesa, de manera transparente y cabal, toda la información referente a producción a fin de compartir sus técnicas, información, fuentes de materiales y recursos, unificar criterios, estandarizar procesos y establecer la forma de producir bajo una misma visión.

En virtud de la dinámica de este sector productivo, se recomienda que se evalúe su situación con cierta frecuencia, antes de tomar futuras acciones de cooperación en la red, en función los cambios que ocurran en el entorno, tomando en cuenta aquellos que podrían tener mayor influencia.

La información resultante de la presente investigación puede servir de base para nuevos estudios dado que el tema cuenta con potencialidad investigativa y aplicativa en la que se podría hacer una dedicación importante. 


\section{Referencias}

Consorcio de Consejos Provinciales del Ecuador (CONCOPE). 2011. Cadenas productivas y desarrollo económico rural en Latinoamérica. Quito: CONCOPE.

Dussel, Enrique. 2006. 20 Tesis de política. Ciudad de México: Siglo XXI / CREFAL.

Galán, José Luis, e Ignacio Castro. 2014. "Las relaciones interorganizativas como fuente de capital social". Universia Business Review, No. 2: 104-117.

Gobierno Provincial de Tungurahua. 2015. Agenda Tungurahua 2015-2017. Consulta: marzo de 2016. 〈https://issuu.com/gobtungurahua/docs/pdf_agenda_tungurahua_20152017_ hgp).

Hendry, Chris, James Brown, Robert DeFillippi y Robert Hassink. 2014. "Industry Clusters as Commercial, Knowledge and Institutional Networks: Opto-electronics in Six Regions in the UK, USA and Germany". En Anna Grandori, edit., Interfirm Networks: Organizational and industrial competitiveness, 151-184. Londres: Routledge.

Instituto Nacional de Estadística y Censos (INEC). 2012. Directorio de empresas y establecimientos. Consulta: abril de 2016. 〈http://www.planificacion.gob.ec/wp-content/uploads/ downloads/2014/02/140210-DirEmpresas-final4.pdf .

---. 2014. Redatam. Consulta: septiembre de 2016. 〈http://redatam.inec.gob.ec/cgibin/RpWeb Engine.exe/EasyCross?\&BASE=DIEE2014\&ITEM=CRUZSIMPLE\&MAIN=WebServerMain.inls.

Jácome, Hugo, y Katiuska King, coord. 2013. Estudios industriales de la micro, pequeña y mediana empresa. Quito: FLACSO / MIPRO. Consulta: febrero de 2016. 〈http://www.industrias.gob.ec/wp-content/uploads/downloads/2013/08/ESTUDIOS_INDUSTRIALES_MI PYMES.pdf).

Liendo, Mónica, y Adriana Martínez. 2001. “Asociatividad: una alternativa para el desarrollo $\mathrm{y}$ crecimiento de las pymes". Instituto de Investigaciones Económicas, Escuela de Economía. Consulta: mayo de 2016. 〈https://www.fcecon.unr.edu.ar/web/sites/default/files/u16/ Decimocuartas/Liendo,\%20Martinez_asociatividad.pdf〉.

Maldovan Bonelli, Johanna, y Nicolás Dzembrowski. 2009. "Asociatividad para el trabajo: una conceptualización de sus dimensiones". Margen: Revista de Trabajo Social, No. 55: $1-9$.

Marchetti, Marcelo. 2008. "Redes estratégicas de pymes: un modelo para mejorar la competitividad". Tesis doctoral en Administración. Universidad Nacional de Rosario, Rosario.

McCormick Escandón, David, y Patricia Morales Ledesma. 2006. "Diferencias conceptuales entre clústers y cadenas productivas y sus implicaciones para la construcción del futuro de Risaralda". Revista Gestión y Región, No. 2: 27-47.

Ministerio Coordinador de Producción, Empleo y Competitividad (MIPRO). 2011. Agendas para la transformación productiva territorial: provincia de Tungurahua. Consulta: septiembre de 2016. 〈http://www.produccion.gob.ec/wp-content/uploads/downloads/2013/02/AGENDA-TERRITORIAL-TUNGURAHUA.pdf〉. 
Naciones Unidas, CEPAL y OCDE. 2012. Perspectivas económicas de América Latina 2013: políticas de PYMES para el cambio estructural. Consulta: abril de 2016. 〈http://www. cepal.org/es/publicaciones/1463-perspectivas-economicas-america-latina-2013-politicas-pymes-cambio-estructural〉.

Navarro Arancegui, Mikel. 2003. “Análisis y políticas de clústers: teoría y realidad”. Ekomiaz: Revista Vasca de Economía, No. 53: 14-49.

Organización Internacional del Trabajo (OIT). 2015. "Pequeñas y medianas empresas y creación de empleo decente y productivo". Informe IV Conferencia Internacional del Trabajo. Consulta: diciembre de 2017. 〈http://www.herramientasoit.org/_lib/file/doc/PyME\%20 -\%20CIT\%202015.pdf .

Pallares Villegas, Zoilo. 2003. "La asociatividad empresarial: una respuesta de los pequeños productores a la internacionalización de las economías”. Hojas Económicas: Revista de la Escuela de Economía, No. 13: 57-74.

Paz y Miño, Juan. 2015. "El socialismo del siglo XXI en Ecuador”. Boletín del The-Taller de Historia Económica. Quito: Pontificia Universidad Católica del Ecuador, Facultad de Economía. Consulta: diciembre de 2017. 〈http://puce.the.pazymino.com/JPyMC SOCIA LISMO_SIGLO_XXI_ECUADOR.pdf».

Pérez Fernández, José. 2015. La regulación financiera: ¿solución o problema? Madrid: Fundación de Estudios Financieros.

Porter, Michael. 1998. Ser competitivos: nuevas aportaciones y conclusiones. Bilbao: Deusto.

Raines, Philip. 2000. "Developing Cluster Policies in Seven European Regions". Regional and Industrial Policy Research Paper, No. 42. Glasgow: European Policies Research Centre / University of Strathclyde.

Rodríguez, Martín Esteban. 2014. “Clústers y aglomerados productivos para el desarrollo regional: el conglomerado metalmecánico de Palpalá”. Ponencia presentada en las III Jornadas Nacionales de "Estudios regionales y mercados de trabajo". San Salvador de Jujuy: Universidad Nacional de Jujuy / Red SIMEL. Consulta: julio de 2016. 〈https:/www.aa cademica.org/iii.jornadas.nacionales.sobre.estudios.regionales.y.mercados.de.trabajo/8. pdf〉.

Valdivia Altamirano, William. 2011. "Asociatividad y competitividad, una aproximación”. Economía Sociedad y Territorio 32, No. 1: 547-552.

Vergara, Sebastián. 2005. "Pequeñas y medianas empresas en América Latina”. Lima: Naciones Unidas / CEPAL. Consulta: enero de 2018. 〈https:/www.researchgate.net/profile/Sebastian_Vergara2/publication/228959619_Pequenas_y_Medianas_Empresas_en_America_Latina/links/55316b3f0cf27acb0dea96a5/Pequenas-y-Medianas-Empresas-en-America-Latina.pdf>.

Ynoub, Roxana. 2015. Cuestión de método:aportes para una metodología crítica. Ciudad de México: Cengage Learning. 


\section{Publicaciones periódicas}

"187 firmas de calzado cierran sus puertas". La Hora (Ambato), 3 de abril de 2016. Consulta: junio de 2016. «ttp://lahora.com.ec/index.php/noticias/show/1101931303\#.WOOvT2-GPIU.

“Artesanos de calzado cierran sus talleres". 2016. La Hora (Ambato), 9 de abril de 2016. Consulta: junio de 2016. «https://issuu.com/la_hora/docs/ambato09abril2016〉.

"La producción de calzado pisa fuerte en el país". 2016. Líderes (Quito). Consulta: septiembre de 2016. 〈http://www.revistalideres.ec/lideres/produccion-calzado-pisa-fuerte-pais.html〉.

Pérez Fernández, Ricardo. 2015. “América Latina ¿El retorno de la derecha, o el surgimiento de la anti-política?". Listin Diario (Santo Domingo), 13 de diciembre de 2015. Consulta: agosto de 2016. 〈http://www.listindiario.com/la-republica/2015/12/13/400209/america-latina-el-retorno-de-la-derecha-o-el-surgimiento-de-la-anti-politica). 